\title{
Retraction Note to: Lepton Mixing and CP Violation Phase in the 3-3-1 Model with Neutral Leptons Based on $T_{13}$ Flavor Symmetry
}

\author{
Vo Van Vien ${ }^{1}$
}

Published online: 1 September 2015

(C) Sociedade Brasileira de Física 2015

Retraction Note to: Braz J Phys (2015) 45:467-480

DOI 10.1007/s13538-015-0330-2

After publication of this paper, it came to our attention that most of the material presented as original research had already been published in another journal.

The Brazilian Journal of Physics has the policy of not publishing articles that contain large portions of previously published material. We consider this a serious breach of the ethics of publication.

In view of this fact, after careful analysis, the editorial board of the Brazilian Journal of Physics has decided to retract this paper.

The online version of the original article can be found at http://dx.doi.org/ $10.1007 / \mathrm{s} 13538-015-0330-2$.

\footnotetext{
Vo Van Vien

wvienk16@gmail.com

1 Department of Physics, Tay Nguyen University, 567 Le Duan, Buon Ma Thuot, DakLak, Vietnam
} 